\title{
Influence of Different Propagation Methods in Vegetative Characters of Some Commercial Varieties of Banana
}

\author{
P. K. Nayak ${ }^{1}$, K. Pavani ${ }^{2}$, V. Divya Vani ${ }^{3}$ and C.M. Panda ${ }^{1}$ \\ ${ }^{1}$ Department of Fruit Science and Horticulture Technology, College of Agriculture, Orissa \\ University of Agriculture and Technology, Bhubaneswar-751003, Odisha, India. \\ ${ }^{2}$ Department of Horticulture, MSSSoA, Centurion University of Technology and Management, \\ Parlakhemundi-761211, Odisha, India \\ ${ }^{3}$ Department of Fruit Science, College of Horticulture, Dr.Y.S.R. Horticultural University, \\ Andhra Pradesh, India, India \\ *Corresponding author
}

\begin{tabular}{|l|}
\hline Ke y w o r d s \\
Grand Naine, \\
Micropropagation, \\
Plant height, \\
Leaves, Shooting \\
\hline Article Info \\
\hline $\begin{array}{l}\text { Accepted: } \\
\text { 20 October } 2020 \\
\text { Available Online: } \\
10 \text { November } 2020\end{array}$ \\
\hline
\end{tabular}

\section{A B S T R A C T}

An experiment entitled Influence of different propagation methods in vegetative characters of some commercial varieties of banana was carried out at the field of the AICRP on Fruits (Banana), at Horticultural Research Station, Orissa University of Agriculture and Technology (OUAT), Bhubaneswar during the year 2017-18. The experiment comprised of twelve (12) treatments replicated thrice and was laid out in a Randomized block design. The 4 varieties of banana viz., Champa (AAB), Bantala (ABB), Patkapura (AAB) and Grand Naine (AAA) together constituted 12 treatments using 3 different propagation methods (Macropropagation, Micropropagation and Sucker propagation). The standard recommended packages of practices were followed in each treatment comprising of 10 plants. Observations in respect to vegetative character of banana were analysed statistically and it was found that micropropagated Grand naine showed maximum girth $(68.82 \mathrm{~cm})$, maximum leaves (43.09) and leaf length along with that it took least number of days for shooting (258.21days), whereas, maximum plant height was attended in micropropagated Bantala $(221.72 \mathrm{~cm})$ and micropropagated champa exhibited maximum leaf width.

\section{Introduction}

Banana (Musa spp.) is the second most important fruit crop in India next to mango. Botanically bananas are the kinds of large herbaceous perennial monocot flowering plants of the genus Musa belonging to the family Musaceae of order Scitamineae. It is a large, perennial monocotyledonous herb. It can grow from 2 to 4 meter in height at maturity. In India, banana is appropriately referred as 'Kalpatharu' a plant of all virtues. 
Each and every part of the plant is used for specific purposes. Apart from its use as a dessert fruit and for culinary purposes, the banana plant has multifaceted uses: the leaf is commonly used as a hygienic dining plate; the male flower is a favourite vegetable; the inner core of the pseudostem is a popular vegetable along with-it banana fibre has multifaceted uses. Banana has emerged as one of the important fruit crops, which is easily available to common man. It is predicted that with everincreasing demand, 60 million tonnes of banana will be needed to meet the domestic demand in 2050. 26 out of 29 Indian states grow bananas. Today, banana is cultivated in more than 130 countries across the world in 5.00 million hectare and yielding 103.63 million tonnes of banana and plantain (FAO, 2013). India is the largest producer of banana in the world with 29.7 million tonnes from an area of 0.88 million hectares with a productivity of $37 \mathrm{MT} / \mathrm{ha}$. Although India accounts for only $15.5 \%$ in area, its contribution in the world's production is $25.58 \%$. Banana is propagated asexually by micropropagation, macropropagation and through suckers. To study the influence of different propagation in banana on its vegetative characters this experiment is carried out.

\section{Materials and Methods}

An experiment entitled "Influence of different propagation methods in vegetative characters of some commercial varieties of banana" was carried out at the field of the AICRP on Fruits (Banana), at Horticultural Research Station, Orissa University of Agriculture and Technology (OUAT), Bhubaneswar during the year 2017-18. The experiment comprised of twelve (12) treatments replicated thrice and was laid out in a Randomized block design. The 4 varieties of banana viz., Champa $(\mathrm{AAB})$, Bantala (ABB), Patkapura (AAB) and
Grand Naine (AAA) together constitute 12 treatments using 3 different propagation methods (Macropropagation (M), Micropropagation (m) and Sucker propagation $(\mathrm{S})$ ). The standard recommended packages of practices were followed in each treatment comprising of 10 plants. Data collected were: plant height and girth, number of functional and total leaves at shooting, number of days to shooting, emergence of sucker and leaf length and width. Functional leaves were considered to be those ones with more than $50 \%$ of their surface area still green. Plant height and girth, leaf length and width were measured using a measuring tape and the respective leaves being tagged, while total leaves were determined by counting all the existing leaves per plant. The suckers were counted manually at different days.

\section{Results and Discussion}

Significantly maximum plant height $(275.12 \mathrm{~cm})$ was recorded in Bantala $(\mathrm{m})$ where as minimum plant height $(221.72 \mathrm{~cm})$ in Grand Naine(S) at shooting. Bantala(m) exhibited highest plant height in all the periodic observations from 30 DAP to 240 DAP. In case of plant girth, Grand Naine(m) exhibited maximum plant girth $(68.82 \mathrm{~cm})$ and minimum plant girth $(58.08 \mathrm{~cm})$ was found in Champa(S). Grand Naine(m) exhibited highest plant girth in all the periodic observations from 30 DAP to 240 DAP. Significantly maximum emergence of suckers (4.85, 4.74, and 5.80) was recorded in Grand Naine(M) at 90 DAP, 120 DAP and 150 DAP followed by Patkapura(M) and minimum emergence of sucker in Champa(m) during all periodical observations. However, at harvesting stage maximum emergence of sucker (6.27) was obtained in Bantala(M) which was found at par with $\operatorname{Bantala}(\mathrm{S})$ and Grand Naine(M) (Table 1-9). 
Table.1 Plant height $(\mathrm{cm})$

\begin{tabular}{|c|c|c|c|c|c|c|c|c|c|}
\hline Treatments & $\begin{array}{l}\text { 30 } \\
\text { DAP }\end{array}$ & $\begin{array}{l}60 \\
\text { DAP }\end{array}$ & $\begin{array}{l}90 \\
\text { DAP }\end{array}$ & $\begin{array}{l}120 \\
\text { DAP }\end{array}$ & $\begin{array}{l}150 \\
\text { DAP }\end{array}$ & $\begin{array}{l}180 \\
\text { DAP }\end{array}$ & $\begin{array}{l}210 \\
\text { DAP }\end{array}$ & $\begin{array}{l}240 \\
\text { DAP }\end{array}$ & $\begin{array}{l}\text { At } \\
\text { shooting } \\
\text { stage }\end{array}$ \\
\hline $\mathrm{T}_{1}$ - Champa (M) & 41.30 & 61.66 & 92.25 & 122.38 & 166.54 & 189.80 & 212.31 & 240.65 & 260.66 \\
\hline $\mathbf{T}_{2-}$ Champa (m) & 43.08 & 63.54 & 96.98 & 129.24 & 173.25 & 197.72 & 221.44 & 242.99 & 264.20 \\
\hline $\mathbf{T}_{3^{-}}$Champa (S) & 40.55 & 60.88 & 91.62 & 121.30 & 164.82 & 187.90 & 210.23 & 238.40 & 259.56 \\
\hline $\mathrm{T}_{4}$ - Bantala $(\mathrm{M})$ & 42.36 & 63.60 & 95.10 & 125.50 & 170.78 & 195.15 & 218.77 & 240.23 & 269.63 \\
\hline $\mathbf{T}_{5}$ - Bantala $(\mathrm{m})$ & 43.65 & 65.01 & 98.84 & 131.34 & 177.03 & 203.17 & 228.56 & 244.11 & 275.12 \\
\hline $\mathbf{T}_{6-}$ Bantala $(\mathrm{S})$ & 41.66 & 62.74 & 94.26 & 124.40 & 168.82 & 192.79 & 216.01 & 235.74 & 266.89 \\
\hline $\mathrm{T}_{7-}$ Patkapura (M) & 37.68 & 59.55 & 89.89 & 120.20 & 164.50 & 187.89 & 210.53 & 230.01 & 250.22 \\
\hline $\mathbf{T}_{8}$ - Patkapura (m) & 38.79 & 61.03 & 92.29 & 124.57 & 168.40 & 192.68 & 216.21 & 237.24 & 256.67 \\
\hline $\mathbf{T}_{9}$ - Patkapura (S) & 37.25 & 59.03 & 89.23 & 119.64 & 162.96 & 185.84 & 207.97 & 227.60 & 249.96 \\
\hline $\mathrm{T}_{10^{-}}$Grand Naine (M) & 34.81 & 54.42 & 81.64 & 112.91 & 157.46 & 181.10 & 200.00 & 214.06 & 222.40 \\
\hline $\mathrm{T}_{11^{-}}$Grand Naine (m) & 36.38 & 56.21 & 82.82 & 117.81 & 162.31 & 187.25 & 204.12 & 215.15 & 226.81 \\
\hline$T_{12-}$ Grand Naine (S) & 34.23 & 53.82 & 80.82 & 112.21 & 156.08 & 179.52 & 197.87 & 211.72 & 221.72 \\
\hline $\operatorname{SEM}( \pm)$ & 0.64 & 1.44 & 1.01 & 0.68 & 0.86 & 1.11 & 1.25 & 0.91 & 0.68 \\
\hline CD (0.05) & 1.88 & 4.26 & 2.98 & 2.02 & 2.55 & 3.26 & 3.70 & 2.67 & 1.99 \\
\hline
\end{tabular}

Table.2 Plant girth $(\mathrm{cm})$

\begin{tabular}{|c|c|c|c|c|c|c|c|c|c|}
\hline Treatments & $\begin{array}{c}30 \\
\text { DAP }\end{array}$ & $\begin{array}{c}60 \\
\text { DAP }\end{array}$ & $\begin{array}{c}90 \\
\text { DAP }\end{array}$ & $\begin{array}{c}120 \\
\text { DAP }\end{array}$ & $\begin{array}{c}150 \\
\text { DAP }\end{array}$ & $\begin{array}{c}180 \\
\text { DAP }\end{array}$ & $\begin{array}{c}210 \\
\text { DAP }\end{array}$ & $\begin{array}{c}\text { 240D } \\
\text { AP }\end{array}$ & $\begin{array}{c}\text { At } \\
\text { shooting } \\
\text { stage }\end{array}$ \\
\hline $\mathrm{T}_{1}$ - Champa (M) & 9.46 & 16.43 & 25.64 & 35.64 & 44.74 & 49.54 & 53.91 & 57.41 & 60.41 \\
\hline $\mathbf{T}_{2^{-}}$Champa (m) & 10.28 & 16.95 & 26.73 & 36.97 & 46.99 & 52.09 & 56.09 & 60.19 & 65.19 \\
\hline $\mathbf{T}_{3^{-}}$Champa $(\mathrm{S})$ & 9.79 & 15.89 & 24.79 & 34.08 & 43.21 & 47.41 & 52.28 & 55.38 & 58.08 \\
\hline $\mathbf{T}_{4}$ - Bantala (M) & 10.29 & 17.37 & 26.58 & 36.58 & 45.68 & 50.81 & 55.51 & 59.01 & 62.01 \\
\hline $\mathbf{T}_{5}$ - Bantala $(\mathrm{m})$ & 10.55 & 18.75 & 28.86 & 39.44 & 48.19 & 53.29 & 58.29 & 62.39 & 67.39 \\
\hline T$_{6}$ - Bantala $(S)$ & 10.32 & 17.25 & 26.15 & 35.44 & 43.94 & 50.08 & 54.88 & 57.98 & 60.68 \\
\hline $\mathrm{T}_{7-}$ Patkapura (M) & 10.16 & 17.06 & 26.27 & 36.27 & 45.37 & 50.17 & 54.87 & 58.37 & 61.37 \\
\hline $\mathbf{T}_{8}$ - Patkapura (m) & 10.45 & 17.95 & 27.06 & 37.64 & 47.06 & 52.16 & 57.16 & 61.26 & 66.26 \\
\hline T9- Patkapura $(\mathrm{S})$ & 10.29 & 16.62 & 25.52 & 34.81 & 43.98 & 48.18 & 53.65 & 56.75 & 59.45 \\
\hline $\mathrm{T}_{10}$-Grand Naine (M) & 10.54 & 18.44 & 27.65 & 37.65 & 46.75 & 51.55 & 56.25 & 59.75 & 62.75 \\
\hline$T_{11}$-Grand Naine (m) & 10.58 & 19.18 & 29.29 & 39.87 & 49.62 & 54.72 & 59.72 & 63.82 & 68.82 \\
\hline $\mathbf{T}_{12}$-Grand Naine (S) & 10.55 & 17.98 & 26.88 & 36.17 & 44.67 & 50.20 & 55.00 & 58.10 & 60.80 \\
\hline $\operatorname{SEM}( \pm)$ & 0.17 & 0.25 & 0.29 & 0.30 & 0.26 & 0.28 & 0.25 & 0.26 & 0.25 \\
\hline CD (0.05) & 0.51 & 0.73 & 0.85 & 0.89 & 0.75 & 0.81 & 0.75 & 0.76 & 0.75 \\
\hline
\end{tabular}


Table.3 Emergence of sucker

\begin{tabular}{|c|c|c|c|c|c|c|c|}
\hline Treatments & $\begin{array}{l}90 \\
\text { DAP }\end{array}$ & $\begin{array}{l}120 \\
\text { DAP }\end{array}$ & $\begin{array}{l}150 \\
\text { DAP }\end{array}$ & $\begin{array}{l}180 \\
\text { DAP }\end{array}$ & $\begin{array}{l}210 \\
\text { DAP }\end{array}$ & $\begin{array}{l}240 \\
\text { DAP }\end{array}$ & $\begin{array}{l}\text { Harvesting } \\
\text { stage }\end{array}$ \\
\hline $\mathbf{T}_{1-}$ Champa (M) & 3.33 & 3.86 & 4.16 & 4.16 & 3.02 & 2.56 & 5.80 \\
\hline $\mathbf{T}_{2^{-}}$Champa (m) & 2.31 & 3.19 & 4.57 & 3.14 & 3.11 & 1.96 & 3.71 \\
\hline $\mathbf{T}_{3^{-}}$Champa (S) & 2.73 & 3.59 & 4.98 & 3.49 & 3.47 & 2.36 & 5.46 \\
\hline $\mathrm{T}_{4}$ - Bantala $(\mathrm{M})$ & 3.62 & 4.63 & 4.11 & 4.21 & 3.76 & 3.28 & 6.27 \\
\hline $\mathbf{T}_{5}$ - Bantala $(\mathrm{m})$ & 3.11 & 4.15 & 5.03 & 4.70 & 3.67 & 2.05 & 4.35 \\
\hline $\mathbf{T}_{6}-$ Bantala $(S)$ & 3.53 & 4.55 & 5.44 & 5.05 & 4.03 & 2.45 & 6.10 \\
\hline $\mathrm{T}_{7-}$ Patkapura (M) & 4.73 & 4.74 & 4.69 & 3.95 & 3.40 & 3.06 & 4.27 \\
\hline T $_{8-}$ Patkapura (m) & 2.94 & 4.15 & 4.25 & 4.32 & 3.28 & 2.96 & 2.12 \\
\hline 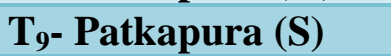 & 3.36 & 4.55 & 4.66 & 4.67 & 3.64 & 3.36 & 3.87 \\
\hline$T_{10}$-Grand Naine (M) & 4.85 & 4.74 & 5.80 & 4.61 & 4.33 & 3.71 & 6.02 \\
\hline $\begin{array}{l}\text { T }_{11^{-}} \text {Grand Naine } \\
(\mathrm{m})\end{array}$ & 3.54 & 4.17 & 5.28 & 3.05 & 3.01 & 2.70 & 2.12 \\
\hline $\mathbf{T}_{12}$ - Grand Naine $(S)$ & 3.96 & 4.57 & 5.69 & 2.38 & 3.37 & 3.10 & 5.21 \\
\hline $\operatorname{SEM}( \pm)$ & 0.11 & 0.12 & 0.16 & 0.32 & 0.12 & 0.09 & 0.13 \\
\hline CD (0.05) & 0.34 & 0.36 & 0.46 & 0.94 & 0.34 & 0.28 & 0.39 \\
\hline
\end{tabular}

Table.4 Total number of leaves produced till flag leaf

\begin{tabular}{|c|c|}
\hline Treatments & Total number of leaves produced \\
\hline $\mathrm{T}_{1}$ - Champa (M) & 40.71 \\
\hline $\mathbf{T}_{2^{-}}$Champa (m) & 42.41 \\
\hline $\mathbf{T}_{3^{-}}$Champa $(\mathbf{S})$ & 40.82 \\
\hline $\mathbf{T}_{4^{-}}$Bantala $(\mathbf{M})$ & 40.28 \\
\hline $\mathbf{T}_{5}-$ Bantala $(\mathrm{m})$ & 41.79 \\
\hline T $_{6}-$ Bantala $(S)$ & 40.20 \\
\hline $\mathbf{T}_{7^{-}}$Patkapura (M) & 40.50 \\
\hline $\mathbf{T}_{8-}$ Patkapura (m) & 40.92 \\
\hline T9- Patkapura (S) & 40.05 \\
\hline $\begin{array}{l}T_{10^{-}} \text {Grand Naine } \\
(\mathrm{M})\end{array}$ & 42.27 \\
\hline$T_{11^{-}}$Grand Naine (m) & 43.09 \\
\hline $\mathbf{T}_{12^{-}}$Grand Naine (S) & 42.06 \\
\hline $\operatorname{SEM}( \pm)$ & 0.10 \\
\hline CD (0.05) & 0.29 \\
\hline
\end{tabular}


Table.5 Number of leaves present at shooting

\begin{tabular}{|c|c|}
\hline Treatments & Number of leaves present at shooting \\
\hline $\mathrm{T}_{1-}$ - Champa $(\mathrm{M})$ & 12.82 \\
\hline $\mathbf{T}_{2-}$ Champa (m) & 13.41 \\
\hline $\mathbf{T}_{3^{-}}$Champa $(S)$ & 12.16 \\
\hline $\mathbf{T}_{4^{-}}$Bantala $(\mathbf{M})$ & 10.90 \\
\hline $\mathbf{T}_{5}$ - Bantala $(\mathrm{m})$ & 11.35 \\
\hline $\mathbf{T}_{6-}$ Bantala $(\mathbf{S})$ & 10.68 \\
\hline $\mathrm{T}_{7-}$ Patkapura (M) & 10.11 \\
\hline $\mathbf{T}_{8^{-}}$Patkapura (m) & 10.86 \\
\hline T9- Patkapura (S) & 9.76 \\
\hline $\begin{array}{l}T_{10^{-}} \text {Grand Naine } \\
(\mathrm{M})\end{array}$ & 12.32 \\
\hline $\mathrm{T}_{11^{-}}$Grand Naine (m) & 12.65 \\
\hline $\mathbf{T}_{12^{-}}$Grand Naine (S) & 11.65 \\
\hline $\operatorname{SEM}( \pm)$ & 0.31 \\
\hline CD (0.05) & 0.93 \\
\hline
\end{tabular}

Table.6 leaf length $(\mathrm{cm})$

\begin{tabular}{|c|c|c|c|c|c|c|c|c|c|}
\hline Treatments & $\begin{array}{l}\text { 30 } \\
\text { DAP }\end{array}$ & $\begin{array}{l}\text { 60 } \\
\text { DAP }\end{array}$ & $\begin{array}{l}90 \\
\text { DAP }\end{array}$ & $\begin{array}{l}120 \\
\text { DAP }\end{array}$ & $\begin{array}{l}150 \\
\text { DAP }\end{array}$ & $\begin{array}{l}180 \\
\text { DAP }\end{array}$ & $\begin{array}{l}210 \\
\text { DAP }\end{array}$ & $\begin{array}{l}240 \\
\text { DAP }\end{array}$ & $\begin{array}{l}\text { At shooting } \\
\text { stage }\end{array}$ \\
\hline $\mathrm{T}_{1}$ - Champa (M) & 32.37 & 51.90 & 70.90 & 88.46 & 104.03 & 119.93 & 129.90 & 139.78 & 151.78 \\
\hline $\mathbf{T}_{2-}$ Champa (m) & 35.07 & 53.40 & 72.53 & 91.90 & 108.93 & 121.96 & 131.96 & 141.99 & 153.99 \\
\hline $\mathbf{T}_{3^{-}}$Champa (S) & 31.65 & 50.65 & 69.11 & 87.14 & 103.18 & 119.21 & 128.87 & 138.83 & 150.83 \\
\hline $\mathbf{T}_{4}$ - Bantala (M) & 37.42 & 50.75 & 63.54 & 80.24 & 95.28 & 109.31 & 119.01 & 128.77 & 140.97 \\
\hline $\mathbf{T}_{5}$ - Bantala (m) & 38.78 & 52.12 & 65.58 & 83.28 & 98.31 & 110.34 & 121.34 & 130.80 & 142.80 \\
\hline $\mathbf{T}_{6}$ - Bantala (S) & 36.56 & 50.23 & 63.36 & 79.63 & 93.89 & 112.16 & 117.63 & 127.88 & 140.18 \\
\hline $\mathbf{T}_{7}$ - Patkapura (M) & 36.85 & 50.18 & 62.98 & 78.68 & 92.38 & 107.08 & 116.74 & 126.70 & 138.90 \\
\hline $\mathrm{T}_{8^{-}}$Patkapura (m) & 37.88 & 51.22 & 64.01 & 81.04 & 96.74 & 109.11 & 119.44 & 129.58 & 141.81 \\
\hline T9- Patkapura (S) & 36.33 & 49.66 & 62.46 & 77.82 & 92.19 & 106.56 & 116.89 & 126.06 & 138.47 \\
\hline $\mathrm{T}_{10^{-}}$Grand Naine (M) & 36.81 & 54.49 & 71.53 & 90.47 & 107.42 & 120.37 & 130.37 & 140.38 & 152.74 \\
\hline $\mathbf{T}_{11^{-}}$Grand Naine (m) & 38.84 & 55.20 & 72.54 & 93.80 & 110.05 & 124.64 & 133.30 & 142.86 & 155.19 \\
\hline $\mathrm{T}_{12-}$ Grand Naine (S) & 36.29 & 53.70 & 70.52 & 89.90 & 106.29 & 119.34 & 129.34 & 139.07 & 151.07 \\
\hline $\operatorname{SEM}( \pm)$ & 0.74 & 0.25 & 0.85 & 0.48 & 1.06 & 1.15 & 0.51 & 0.44 & 0.40 \\
\hline CD (0.05) & 2.17 & 0.73 & 2.50 & 1.42 & 3.12 & 3.40 & 1.50 & 1.31 & 1.17 \\
\hline
\end{tabular}


Table.7 Leaf width $(\mathrm{cm})$

\begin{tabular}{|c|c|c|c|c|c|c|c|c|c|}
\hline Treatments & $\begin{array}{l}\text { 30 } \\
\text { DAP }\end{array}$ & $\begin{array}{l}\text { 60 } \\
\text { DAP }\end{array}$ & $\begin{array}{l}90 \\
\text { DAP }\end{array}$ & $\begin{array}{l}120 \\
\text { DAP }\end{array}$ & $\begin{array}{l}150 \\
\text { DAP }\end{array}$ & $\begin{array}{l}180 \\
\text { DAP }\end{array}$ & $\begin{array}{l}210 \\
\text { DAP }\end{array}$ & $\begin{array}{l}240 \\
\text { DAP }\end{array}$ & $\begin{array}{l}\text { At } \\
\text { shooting } \\
\text { stage }\end{array}$ \\
\hline $\mathrm{T}_{1}$ - Champa (M) & 13.76 & 17.50 & 21.60 & 28.83 & 35.13 & 41.76 & 50.06 & 54.26 & 60.01 \\
\hline $\mathbf{T}_{2}$ - Champa (m) & 14.83 & 18.23 & 22.33 & 29.56 & 36.86 & 43.16 & 51.99 & 56.84 & 62.37 \\
\hline$T_{3}-$ Champa (S) & 13.43 & 17.12 & 21.22 & 28.45 & 34.75 & 41.35 & 49.83 & 53.97 & 59.17 \\
\hline $\mathbf{T}_{4}$ - Bantala (M) & 12.57 & 16.57 & 20.67 & 27.90 & 34.20 & 40.50 & 48.71 & 53.29 & 58.79 \\
\hline $\mathbf{T}_{5}-$ Bantala (m) & 13.64 & 17.34 & 21.44 & 28.83 & 34.47 & 40.77 & 49.57 & 54.77 & 59.82 \\
\hline $\mathbf{T}_{6}-$ Bantala $(\mathbf{S})$ & 12.23 & 16.25 & 20.35 & 27.24 & 33.54 & 39.84 & 48.09 & 52.96 & 58.09 \\
\hline $\mathbf{T}_{7}$ - Patkapura (M) & 12.23 & 16.23 & 20.33 & 27.56 & 33.86 & 39.50 & 47.63 & 52.71 & 57.76 \\
\hline $\mathbf{T}_{8-}$ Patkapura (m) & 13.23 & 17.16 & 21.26 & 27.82 & 34.12 & 40.02 & 48.89 & 53.77 & 58.74 \\
\hline T9- Patkapura (S) & 11.84 & 15.87 & 19.97 & 27.20 & 33.50 & 39.14 & 47.23 & 52.06 & 57.23 \\
\hline $\mathrm{T}_{10^{-}}$Grand Naine (M) & 13.57 & 17.17 & 21.27 & 28.50 & 35.03 & 41.33 & 49.27 & 54.34 & 59.80 \\
\hline $\mathbf{T}_{11^{-}}$Grand Naine (m) & 14.64 & 17.57 & 21.67 & 28.90 & 35.87 & 42.17 & 50.38 & 55.52 & 60.76 \\
\hline $\mathbf{T}_{12^{-}}$Grand Naine (S) & 13.29 & 16.62 & 20.72 & 27.95 & 34.92 & 41.22 & 48.90 & 53.74 & 58.83 \\
\hline $\operatorname{SEM}( \pm)$ & 0.22 & 0.22 & 0.22 & 0.21 & 0.18 & 0.26 & 0.27 & 0.31 & 0.19 \\
\hline CD (0.05) & 0.65 & 0.63 & 0.63 & 0.61 & 0.53 & 0.77 & 0.80 & 0.92 & 0.57 \\
\hline
\end{tabular}

Table.8 No of days taken for shooting

\begin{tabular}{|c|c|}
\hline Treatments & No of days taken for shooting \\
\hline$T_{1}$ - Champa (M) & 289.58 \\
\hline $\mathbf{T}_{2}$ - Champa (m) & 288.48 \\
\hline $\mathbf{T}_{3^{-}}$Champa (S) & 299.15 \\
\hline$T_{4}-$ Bantala $(M)$ & 275.21 \\
\hline $\mathbf{T}_{5}$ - Bantala (m) & 273.00 \\
\hline $\mathbf{T}_{6^{-}}$Bantala $(\mathbf{S})$ & 284.22 \\
\hline $\mathbf{T}_{7}$ - Patkapura (M) & 307.63 \\
\hline $\mathbf{T}_{8}$ - Patkapura (m) & 299.67 \\
\hline $\mathbf{T}_{9}$ - Patkapura (S) & 308.91 \\
\hline $\mathrm{T}_{10^{-}}$Grand Naine (M) & 260.89 \\
\hline $\mathbf{T}_{11^{-}}$Grand Naine (m) & 258.21 \\
\hline$T_{12^{-}}$Grand Naine $(S)$ & 265.49 \\
\hline $\operatorname{SEM}( \pm)$ & 1.19 \\
\hline CD (0.05) & 3.52 \\
\hline
\end{tabular}


The minimum emergence of suckers (2.12) was obtained in Patkapura(m). The number of leaves produced till flag leaf ranged from 40.05to 43.09. Highest number of leaves produced in Grand Naine $(m)$ (43.09) followed by Champa(M) and Grand Naine(M) which were at par with each other. Patkapura (S) recorded the lowest value (40.05).

Maximum number of leaves present at shooting (13.41) was observed in Champa (M) followed by Champa (M) (12.82) and Grand Naine (m) (12.65) which were at par with each other. The lowest number of leaves at shooting (9.76) was found in treatment Patkapura (S). The leaf length of Grand Naine (m) exhibited maximum value $(38.84 \mathrm{~cm}$, $55.20 \mathrm{~cm}, \quad 72.54 \mathrm{~cm}, 93.80 \mathrm{~cm}, 110.05 \mathrm{~cm}$, $124.64 \mathrm{~cm}, \quad 133.30 \mathrm{~cm}, \quad 139.07 \mathrm{~cm}$ and $155.19 \mathrm{~cm})$ at 30 DAP, 60 DAP, 90 DAP, 120 DAP, 150 DAP, 180 DAP, 210 DAP, 240 DAP and shooting stage and showed significant results. The width of leaf of Champa(m) exhibited maximum value $(14.64 \mathrm{~cm}, \quad 18.23 \mathrm{~cm}, 22.33 \mathrm{~cm}, 29.56 \mathrm{~cm}$, $36.86 \mathrm{~cm}, 43.16 \mathrm{~cm}, 51.99 \mathrm{~cm}, 56.84 \mathrm{~cm}$ and $62.37 \mathrm{~cm}$ ) at $30 \mathrm{DAP}, 60 \mathrm{DAP}, 90 \mathrm{DAP}, 120$ DAP, 150 DAP, 180 DAP, 210 DAP, 240 DAP and shooting stage showed significant results. The minimum number of days taken for shooting (258.21days) was found in Grand Naine(m) and Grand Naine(S) (260.89days) which were statistically at par with each other and highest number of days for shooting (308.91days) was taken by Patkapura(S) followed by Patkapura(M) (307.63days) and both were statistically at par with each other.

In conclusion, the results revealed that none of the genotypes were found superior for all traits studied. However the treatment Banatala micropropagated recorded maximum plant height $(275.12 \mathrm{~cm})$ while Grand Naine sucker propagated recorded minimum plant height $(221.72 \mathrm{~cm})$. The probable reason may be due to the resultant effect of the genetic makeup of the variety interacted with the environment.
So far as the effects of methods of propagation on plant height is concerned almost all the genotypes produced more height in micropropagated planting in comparison to macropropagation and suckers. This may be due to the ageing effect as the formers are more aged as they are planted maintaining at 45 days from poly nursery. This corroborates the finding of Wong et al., (2002), Singh et al., (2011). maximum emergence of suckers $(4.85,4.74$, and 5.80) was recorded in Grand Naine macropropagated at 90 DAP, 120 DAP and 150 DAP followed by Patkapura macropropagated and minimum emergence of sucker in Champa micropropagated during all periodical observations. However, at harvesting stage maximum emergence of sucker (6.27) was obtained in Bantala macropropagated. The maximum sucker emergence might be due to presence of well developed rhizome in macropropagation and sucker propagation while micropropagation shows minimum emergence of suckers due to the slow healing of the wounded rhizome. Variations in total number of suckers produced in different varieties were earlier reported by Venkataramani (1946) as a varietal difference. Maximum number of leaves till flag leaf (43.09) was recorded in Grand Naine micropropagated. However there is no difference among the propagating methods adopted confirming the assumption. The maximum leaf length was obtained from Grand Naine $(155.19 \mathrm{~cm})$. Grand Naine consumed the minimum time period for shooting (258.21 days) and Patkapura consumed highest (307.63days). This indicates the earliness of the variety which is fully controlled by the inherent genetic makeup. This agrees with the findings of Joab (2014) and Ogero (2012).

\section{References}

Joab V. Characterization of plantain and banana grown in the southern highlands 
of Tanzania. A special project submitted in partial fulfilment of the requirement for the degree of Bachelor of Science in horticulture, Sokoine University of Agriculture, Morogoro, Tanzania. 2004; 17-19.

Ogero KO. 2012. Low cost tissue culture of selected cassava (Manihotes culenta Crantz) and sweet potato (Ipomea batatas (L)Lam.) varieties. M.Sc Thesis, Department of Agricultural Sciences and Technology, Kenyatta University, Nairobi, Kenya.

Singh H.P, S. Uma, R. Selvarajan. J. L. and Karihalo. (2011). Micropropagation for
Production of Quality Banana Planting Materials in Asian Pacific. . Asian Pacific Consortium on Agricultural Biotechnology (APCoAB). New Delhi, India., 92.

Venkataramani KS.1946. Studies on Indian bananas. A descriptive study of twenty fourvarieties. Proc. Indian Acad. Sci. Soc., 23: 113-128.

Wong C, Kiew R, Argent G, Set O, Lee SK, Gan YY. 2002. Assessment of the Validity of the Sections in Musa (Musaceae) using AFLP, Annals of Botany, Volume 90, Issue 2, 1, Pages 231-238.

\section{How to cite this article:}

Nayak, P.K., K. Pavani, V. Divya Vani and Panda, C.M. 2020. Influence of Different Propagation Methods in Vegetative Characters of Some Commercial Varieties of Banana. Int.J.Curr.Microbiol.App.Sci. 9(11): 2904-2911. doi: https://doi.org/10.20546/ijcmas.2020.911.352 